\title{
Metabolic Risk Markers in Insulin Resistance and Non-Insulin Resistance Type 2 Diabetes Mellitus
}

\author{
Thae Nu Htwe, ${ }^{1 *}$ Ohnmar Myint Thein, ${ }^{2}$ Saw Wut Hmon, ${ }^{3}$ Myat Mon Oo, ${ }^{4}$ Moh Moh Htun, ${ }^{4}$ Myat Thandar \\ ${ }^{1}$ Physiology Department, University of Medicine, Taunggyi \\ ${ }^{2}$ Physiology Department, University of Medicine, Mandalay \\ ${ }^{3}$ Pathology Department, University of Medicine \\ ${ }^{4}$ Research Scientist, Department of Medical Research \\ ${ }^{4}$ Director, Department of Medical Research \\ ${ }^{5}$ Rector (Retired), University of Nursing, Yangon
}

\begin{abstract}
Aim: Type 2 diabetes mellitus (T2DM) is the most common metabolic disorder and its pathogenesis is characterized by a combination of peripheral insulin resistance and impaired insulin secretary capacity of pancreatic $\beta$ cell. Over the years, there has been increasing deaths from T2DM. In Myanmar, there is little information on its causes, due to few published data on the prevalence of MS and its association with T2DM. This study aims at identifying the metabolic risk markers leading to MS in T2DM, as well as the impact of MS on the insulin resistance.

Methods: Hundred T2DM patients were recruited from Diabetic Clinic, Yangon General Hospital. The clinical evaluation consisted of waist circumference, blood pressure, height and weight measurements; the biochemical analysis included determination of fasting plasma glucose, serum insulin and fasting lipid profile. Plasma glucose level was determined by the glucose oxidase method and fasting serum insulin was measured by enzyme linked immunoassay (ELISA) kit method. Insulin resistance (HOMA IR) was calculated using formula by Matthews et al in 1985. Metabolic syndrome was defined as International Diabetes Federation (IDF) criteria.

Results: In the present study, MS was not significantly associated with insulin resistance $(84.72 \%$ in the insulin resistance group vs $75 \%$ in the non-insulin resistance group). There were no significant differences in metabolic risk markers between the insulin resistance and non- insulin resistance groups. Present study showed $80 \%$ of insulin resistance male patients and $85 \%$ of insulin resistance female patients had MS, and also $83 \%$ of non-insulin resistance male patients and that of female patients had $64 \%$ of MS, respectively. There was no significant association between each group.
\end{abstract}

Conclusion: Metabolic syndrome was found in 83 patients in the present study, and of which 61 patients were found to show insulin resistance. Metabolic syndrome was not significantly associated with presence or absence of insulin resistance.

Keywords: Insulin resistance, Metabolic syndrome, Metabolic risk markers, Type 2 diabetes mellitus

\section{Introduction}

Obesity, in particular excess visceral adiposity, is associated with insulin resistance (IR), hyperglycaemia, dyslipidaemia and hypertension, which together are termed "metabolic syndrome" (MS). These metabolic disorders increase the risk of development of type 2 diabetes mellitus (T2DM) and cardiovascular diseases and contribute to high rates of mortality and morbidity. ${ }^{1}$ Insulin resistance-linked obesity is caused by poor dieting and lack of regular exercise. Other genetic or lifestyle risk factors lead to the MS.

Association between insulin resistance and lipid parameters is

\begin{tabular}{|l|l|}
\hline \hline Quick Response Code: & *Corresponding author: Thae Nu Htwe, Professor and Head, Physiology Department, Univer- \\
sity of Medicine, Tel +9595044707, Taunggyi, Myanmar \\
Received: 28 August, 2021 \\
Citation: Htwe TN, Thein OM, Hmon SW, Mon Oo M, Htun MM, et al. Metabolic Risk Markers \\
in Insulin Resistance and Non-Insulin Resistance Type 2 Diabetes Mellitus. SOJ Diabet Endocr \\
Care. 2021;1(2):1-4. DOI: 10.53902/SOJDEC.2021.01.000508
\end{tabular}


also conflicting. In Campos's study, ${ }^{2}$ HOMA-IR was not associated with TC, TG, HDL and LDL cholesterol levels. However, Baroni ${ }^{3}$ reported that insulin resistance is generally associated with increased triglyceride concentrations. Fasting insulin and HOMA-IR were inversely associated with HDL cholesterol in some population and reduced HDL cholesterol levels are a feature of insulin resistance. ${ }^{4}$

According to WHO estimation, the prevalence of diabetes mellitus in Myanmar was 2.4\% in 1995 and it will be 3.2\% in the year $2025 .{ }^{5}$ Over the years, there has been increasing deaths from T2DM. Locally, there is little information on its causes, due to few published data on the prevalence of MS and its association with T2DM. This study aims at identifying the metabolic risk markers leading to MS in T2DM, as well as the impact of MS on insulin resistance.

\section{Materials and Methods}

Hundred T2DM patients were recruited from Diabetic Clinic, Yangon General Hospital. The clinical evaluation consisted of waist circumference, blood pressure, height and weight measurements; the biochemical analysis included determination of fasting plasma glucose, serum insulin and fasting lipid profile. Plasma glucose level was determined by the glucose oxidase method and fasting serum insulin was measured by enzyme linked immunoassay (ELISA) kit method. Homeostasis model assessment index for insulin resistance (HOMA IR) was calculated using formula by Matthews ${ }^{6}$ in 1985.0verweight and obesity were defined according to WHO guideline, ${ }^{7}$ overweight as $\mathrm{BMI} \geq 25 \mathrm{~kg} / \mathrm{m}^{2}$, obesity as $\mathrm{BMI} \geq 30 \mathrm{~kg} / \mathrm{m}^{2}$. Metabolic syndrome was defined as International Diabetes Federation (IDF) criteria. ${ }^{8}$ Data were analysed by SPSS (version 16.0) statistical software. Data were presented as mean value \pm standard deviation (SD) and analysed by Fisher exact test, the difference was considered significant when $p$ value is $<0.05$.

\section{Results}

Metabolic risk markers in insulin resistance and non-insulin resistance groups. There were no significant differences in metabolic risk markers between the insulin resistance and non-insulin resistance groups (Table 1).

\section{Metabolic syndrome distribution in insulin resistance and non insulin-resistance T2DM}

Metabolic syndrome was found in 83 patients in the present study, and of which 61 patients was found to show insulin resistance. Metabolic syndrome was not significantly associated with presence or absence of insulin resistance. In the present study, both insulin resistance and non-insulin resistance group, between 40$50 \%$ were found to have 4 components of metabolic syndrome, followed by over $35 \%$ of the study group having all metabolic components. Those without metabolic syndrome were 11 and 6 in insulin resistance and non-insulin resistance group, although there were only one each in both group who has only 2 metabolic components. (Table 2)

\section{Metabolic components in insulin resistance and non- in- sulin resistance groups without metabolic syndrome}

Table 3 shows that 3 metabolic risk markers are present in 8 patients without MS and in 7 patients, 4 metabolic risk markers are present. These data suggest that other metabolic risk parameters could be present without the central obesity.

Table 1: Metabolic risk markers in insulin resistance and non-insulin-resistance groups.

\begin{tabular}{|l|l|l|l|}
\hline \multicolumn{1}{|c|}{ Metabolic Risk Markers } & \multicolumn{1}{|c|}{ IR+mean \pm SD (n=72) } & \multicolumn{1}{c|}{ IR-mean \pm SD (n=28) } & \multicolumn{1}{c|}{ Remark } \\
\hline Waist circumference (cm) & $94.37 \pm 12.31$ & $95.42 \pm 13.39$ & NS \\
\hline Systolic blood pressure (mmHg) & $136.25 \pm 20.49$ & $134.57 \pm 21.15$ & NS \\
\hline Diastolic blood pressure (mmHg) & $82.43 \pm 10.34$ & $82.83 \pm 8.09$ & NS \\
\hline Fasting blood sugar (mg/dL) & $153.65 \pm 66.25$ & $129.39 \pm 40$ & NS \\
\hline Triglyceride (mg/dL) & $187.65 \pm 36.05$ & $193.43 \pm 43.71$ & NS \\
\hline HDL $(\mathrm{mg} / \mathrm{dL})$ & $37.95 \pm 7.64$ & $36.65 \pm 8.48$ & NS \\
\hline
\end{tabular}

NS: not significant

Table 2: Distribution of metabolic syndrome and its components in insulin resistance and non- insulin resistance T2DM.

\begin{tabular}{|l|l|l|l|}
\hline \multicolumn{1}{|c|}{ Metabolic syndrome } & \multicolumn{1}{|c|}{$\begin{array}{c}\text { IR+(n=72) } \\
\text { Number (\%) }\end{array}$} & \multicolumn{1}{c|}{$\begin{array}{c}\text { IR-(n=28) } \\
\text { Number (\%) }\end{array}$} & \multicolumn{1}{c|}{ Remark } \\
\hline Presence & $61(84.72 \%)$ & $22(75 \%)$ & NS \\
\hline Absence & $11(15.27 \%)$ & $6(25 \%)$ & NS \\
\hline 2 components & $1(1.38 \%)$ & $1(3.57 \%)$ & NS \\
\hline 3 components & $12(16.66 \%)$ & $3(10.71 \%)$ & NS \\
\hline 4 components & $30(41.66 \%)$ & $15(53.57 \%)$ & NS \\
\hline 5 components & $29(40.27 \%)$ & $9(32.14 \%)$ & NS \\
\hline
\end{tabular}

NS: not significant 
Metabolic syndrome and metabolic components in insulin resistance and non-insulin resistance male and female patients

Table 4 showed $80 \%$ of insulin resistance male patients and $85 \%$ of insulin resistance female patients had MS, and also $83 \%$ of non-insulin resistance male patients and that of female patients had $81 \%$ of MS, respectively. There was no significant association between each group.

Table 4 Metabolic syndrome and metabolic components in insulin resistance and non-insulin resistance male and female patients.

Table 3: Metabolic components in insulin resistance and non insulin resistance groups without metabolic syndrome.

\begin{tabular}{|l|l|l|}
\hline \multicolumn{1}{|c|}{ Components } & \multicolumn{1}{c|}{ IR+, MS-(n=11) } & \multicolumn{1}{c|}{ IR-, MS-(n=6) } \\
\hline 2 components & 1 & 1 \\
\hline 3 components & 6 & 2 \\
\hline 4 components & 4 & 3 \\
\hline
\end{tabular}

Table 4: Metabolic syndrome and metabolic components in insulin resistance and non-insulin resistance male and female patients.

\begin{tabular}{|l|l|l|l|l|}
\hline \multicolumn{1}{|c|}{ Metabolic syndrome } & $\begin{array}{c}\text { IR+Male (n=25) } \\
\text { Number (\%) }\end{array}$ & $\begin{array}{c}\text { IR+Female (n=52) } \\
\text { Number (\%) }\end{array}$ & $\begin{array}{c}\text { IR-Male (n=12) } \\
\text { Number (\%) }\end{array}$ & $\begin{array}{c}\text { IR-Female (n=11) } \\
\text { Number (\%) }\end{array}$ \\
\hline Presence & $20(80.00)$ & $44(84.62)$ & $10(83.33)$ & $9(81.81)$ \\
\hline Absence & $5(20.00)$ & $8(15.38)$ & $2(16.67)$ & $2(18.18)$ \\
\hline 1 component & $1(4.00)$ & $0(0.00)$ & $0(0.00)$ & $0(0.00)$ \\
\hline 2 components & $1(4.00)$ & $1(1.92)$ & $1(8.33)$ & $0(0.00)$ \\
\hline 3 components & $9(36.00)$ & $11(21.15)$ & $1(8.33)$ & $3(27.27)$ \\
\hline 4 components & $9(36.00)$ & $23(44.23)$ & $7(58.33)$ & $5(45.45)$ \\
\hline 5 components & $5(20.00)$ & $17(32.69)$ & $3(25.00)$ & $3(27.27)$ \\
\hline
\end{tabular}

\section{Discussion}

\section{Metabolic syndrome in type-2 diabetes mellitus}

The prevalence of MS seems to vary among different study population based on the presence of risk factors including BMI, life styles, ethnicity, race, age and sex. It was $83 \%$ in the present study, 85\% in Scott et al study (2011), ${ }^{8} 59.5 \%$ in Ranjith, ${ }^{9} 54.2 \%$ in Rojas, ${ }^{10} 64.6 \%$ in Chung Hua. ${ }^{11}$

The prevalence of MS was found varied when the criteria used for the diagnosis for MS were different. Using the clinical definitions, namely the original NCEP-ATP III, the prevalence of MS in the Philippines in 2003 was $11.9 \%$. It became $18.6 \%$ when the modified AHA/NHLBI criteria were used. ${ }^{12}$ Similarly, the prevalence of MS as defined by the NCEP ATP III criteria was $60.4 \%$ whereas it was close to it, but not exactly the same, $59.5 \%$ in young Indian patients when the IDF criteria were used. ${ }^{9}$

In the present study, MS was not significantly associated with insulin resistance $(84.72 \%$ in the insulin resistance group vs $75 \%$ in the non-insulin resistance group). Such absence of association between MS and insulin resistance was reported also by Ranjith ${ }^{9}$ in 2008. However MS was not significantly associated with presence or absence of insulin resistance, the components of MS in insulin resistance group was higher than non-insulin resistance group (Table 2) but not reached significant level. The limitation of the present study is sample size population, the present study participated only hundred T2DM subjects.
Metabolic risk components such as WC, FBS, and lipid parameters were not significantly different between insulin resistance and non-insulin resistance patients (Table 1), and that finding was in consistence with the finding of Garg study ${ }^{13}$ in 2011 and Khin Saw Than ${ }^{14}$ in 2012 except that in the latter study, WC and FBS were significantly higher in insulin resistance than in non-insulin resistance patients. In the present study FBS level was higher in insulin resistance group than non- insulin resistance group (153.65 $\pm 66.25 v s 129.39 \pm 40)$, but that value does not reached significant level which may be due to inclusion criteria of the study. The present study included overweight and obese T2DM who were taking metformin drug only.

Regarding the components of MS, $45 \%$ were found to have 4 components, followed by $38 \%$ having all metabolic components in (Table 2). The central obesity was not present in some cases despite the presence of other metabolic risk parameters because their BMI are not more 26 and overweight patients. Those without MS were 11 and 6 in insulin resistance and non-insulin resistance group, respectively. There was only one each in both groups who has only 2 metabolic components (Table 3). Although the patients without MS, most of the patients had 3 or 4 metabolic components because the study included overweight and obese patients.

Nsiah $\mathrm{k}^{15}$ showed higher prevalence of MS in female (77.01\%) than male subjects (22.99\%), which was not consistent with the present study, $80 \%$ of insulin resistance male patients and $85 \%$ of insulin resistance female patients had MS, and also $83 \%$ of non-in- 
sulin resistance male patients and that of female patients had $81 \%$ of MS, respectively. The reason may be due to a relatively sedentary lifestyle of the patients in the present study most of them are traders or unemployed.

The present study has shown an increased prevalence of MS (83\%), the most prevalent component was hypertension, followed by central obesity, low HDL-C and hypertriglyceridemia. Low educational status and obesity also have great predictive effects on MS in the type 2 diabetics.

\section{Conclusion}

Metabolic syndrome was found in 83 patients in the present study, and of which 61 patients were found to show insulin resistance. Metabolic syndrome was not significantly associated with presence or absence of insulin resistance and also metabolic risk markers were not significantly associated with presence or absence of insulin resistance.

\section{Limitation of the Study}

One of the limitation is all the patients were recruited from Diabetes Clinic, Yangon General Hospital, so all patients received lipid lowering agents from clinic, actually when the present study considered metabolic components, we need to exclude the patients without taking lipid lowering agents. Second one is small sample size, we can do only 100 patients.

\section{Acknowledgments}

I would like to express my special thanks of gratitude to Professor Myat Thandar (Professor, Medical Education Centre, School of Medicine, International University of Health and Welfare, Chiba, Japan) for her guidance, encouragement and invaluable suggestions.

\section{Funding}

This work was not supported by research grants.

\section{Conflicts of Interest}

The authors declare no conflicts of interest.

\section{References}

1. Alberti KG, Eckel RH, Grundy SM, et al. Harmonizing the metabolic syndrome: a joint interim statement of the International Diabetes Federa- tion Task Force on Epidemiology and Prevention; National heart, lung, and blood institute; American Heart Association; World heart federation; international atherosclerosis society; and international association for the study of obesity. Circulation. 2009;120(6):1640-1645.

2. Campos G, Fernandez V, Fernadez E, et al. Association of free fatty acids with the insulin-resistant state but not with central obesity in individuals from Venezuela. Invest Clin. 2010;51(1):115-126.

3. Baroni MG, Leonettif F, Sentinellif F, et al. The G972R variant of the insulin receptor substrate-1(IRS-1) gene is associated with insulin resistance in uncomplicated obese subjects evaluated by hyperinsulinemic-euglycemic clamp. J Endocrinol Invest. 2004; 27(8):754-759.

4. Howard BV. Insulin resistance and lipid metabolism. Am J Cardiol. 1999;84(1A):28J-32J.

5. World Health Organization, country office for Myanmar (2008) WHO country co-operation strategy 2008-201-Myanmar. New Delhi.

6. Matthews KA, Hosker JP, Rudenski AS, et al. Homeostasis model ascessment: insulin resistance and $\beta$-cell function from fasting plasma glucose and insulin concentration in man. Diabetologia. 1985;28(7):412-419.

7. World Health Organization (2006) Obesity and overweight. Fact sheet No.311, September.

8. Scott R, Donoghoe M, Watts GF, et al. Impact of metabolic syndrome and its components on cardiovascular disease event rates in $4900 \mathrm{pa}-$ tients with type- 2 diabetes assigned to placebo in the field randomized trial. Cardiovasc Diabeto. 2011;10:102-109.

9. Ranjith N, Pegoraro RJ, Naidoo DP, et al. Genetic variants associated with insulin resistance and metabolic syndrome in young Asia Indians with myocardial infarction. Metab Syndr Relat Disord. 2008;6(3):209214.

10. Rojas R, Aguilar Salinas CA, Jimenez Corona A, et al .Metabolic syndrome in Mexican adults. Results from the national health and nutrition survey 2006. Salud Publica Mex. 2010;52(Suppl 1):S11-S18.

11. Chung Hua H. Different impacts of metabolic syndrome components on insulin resistance in type-2 diabetes. International Journal of Endocrinology. 2013.

12. Morales DD, Punzalan FE, Paz pacheco E, et al. Metabolic syndrome in the Philippine general population: prevalence and risk for atherosclerotic cardiovascular disease and diabetes mellitus. Diab Vasc Dis Res. 2008;5(1):36-43.

13. Garg MK, Dutta MK, Mahalle N. Study of beta-cell function (by HOMA model) in metabolic syndrome. Indian J Endocrinol Metab. 2011;15(Suppl 1):S44-S49.

14. Khin Saw Than. Insulin resistance and high sensitive $C$-reactive protein in type-2 diabetic patients. 2012; $\mathrm{PhD}$ thesis. University of Medicine 1 , Yangon.

15. Nsiah K, Owusua Shang V, Agyenim Boateng K. Prevalence of metabolic syndrome in type 2 diabetes mellitus patients. Int J Appl Basic Med Res. 2015;5(2):133-138. 